\title{
Une analyse critériée des besoins linguistiques dans l'enseignement universitaire des Sciences économiques
}

Gail Taillefer

\section{(2) OpenEdition}

Journals

Édition électronique

URL : http://journals.openedition.org/asp/1095

DOI : 10.4000/asp.1095

ISBN : 978-2-8218-0396-1

ISSN : 2108-6354

\section{Éditeur}

Groupe d'étude et de recherche en anglais de spécialité

Édition imprimée

Date de publication : 1 mars 2004

Pagination : 107-124

ISSN : 1246-8185

Référence électronique

Gail Taillefer, « Une analyse critériée des besoins linguistiques dans l'enseignement universitaire des Sciences économiques », ASp [En ligne], 43-44 | 2004, mis en ligne le 01 décembre 2004, consulté le 30 avril 2019. URL : http://journals.openedition.org/asp/1095 ; DOI : 10.4000/asp.1095

Ce document a été généré automatiquement le 30 avril 2019.

Tous droits réservés 


\title{
Une analyse critériée des besoins linguistiques dans l'enseignement universitaire des Sciences économiques
}

\author{
Gail Taillefer
}

\section{Introduction}

1 Le XXVe colloque du GERAS avait pour thème la langue de spécialité et les milieux professionnels ; une des pistes de recherche annoncées était la demande normative de ces milieux à l'égard de la langue et des langues. Mais parallèlement à la demande normative des milieux professionnels se pose celle de la demande critériée : quels niveaux de compétences linguistiques sont nécessaires, voire exigés pour quels milieux professionnels? Comment les formations en langues de spécialité doivent-elles répondre à cette demande?

2 Au printemps 2002, dans le cadre de l'espace européen d'enseignement supérieur prévoyant l'harmonisation des niveaux diplômants, le ministère de l'Éducation nationale a mis en place le «LMD», ou "licence-master-doctorat», correspondant aux grades délivrés après trois, cinq et huit années réussies d'études supérieures. Les dispositions du nouveau diplôme national de master ${ }^{1}$ précisent dans l'article 6 que « le diplôme de master ne peut être délivré qu'après validation de l'aptitude à maîtriser au moins une langue vivante étrangère", et que "les parcours types de formation comprennent des enseignements permettant aux étudiants d'acquérir cette aptitude ». En septembre 2003, l'Observatoire de la vie étudiante de l'université des Sciences sociales où nous travaillons a publié les résultats d'une enquête sur le devenir des maîtres de l'année 1998 (« dernière enquête avant le LMD »)2. Une des questions portait sur l'importance de la maîtrise d'une ou de plusieurs langues étrangères dans l'insertion professionnelle. Si seulement $9 \%$ des 
étudiants en Administration économique et sociale ont répondu qu'une solide compétence en langues a favorisé leur première embauche $(22 \%$ en Droit, $25 \%$ en Gestion), $33 \%$ des diplômés de la maîtrise en Sciences économiques ont répondu affirmativement.

Dans ce contexte de nouvelle exigence nationale et d'importance non négligeable accordée par les diplômés d'une Faculté mondialement reconnue ${ }^{3}$ à une compétence élevée en langues, nous avons voulu cerner cette notion de "maîtrise " pour que l'offre d'enseignement en amont du master réponde à la demande. Plusieurs années au contact des étudiants en Sciences économiques de cette université nous laissent percevoir un sentiment d'insatisfaction par rapport à la formation proposée en langues aussi bien chez les étudiants que chez les enseignants de cette discipline. Depuis les premières années du DEUG (Diplôme d'études universitaires générales, crée en 1973), aucune réflexion de fond sur l'apprentissage, et donc sur l'enseignement, n'a été engagée. Avec le soutien du Doyen de la Faculté des Sciences économiques, une analyse des besoins a donc été conçue pour cerner la question, en sondant les différentes représentations en matière de langues étrangères chez les divers acteurs de la communauté universitaire.

\section{Expérimentation}

\subsection{Cadre théorique}

4 D'abord, que veut-on dire par "besoins » en langues étrangères? T. Hutchinson et A. Waters (1987: 54-63) distinguent des besoins « cibles » des besoins « d'apprentissage ». Les premiers recouvrent les exigences objectives, les perceptions subjectives de ces exigences chez les apprenants, et le "manque à gagner» entre les compétences nécessaires et celles (réelles) des apprenants ; les seconds, les perceptions des apprenants de ce qu'il leur faut pour satisfaire les besoins cibles. T. Dudley-Evans \& M. J. St. John (1998: 121-128) en parlent en termes similaires : « product-oriented target-situation analysis " (besoins réels et perçus), "processus-oriented learning situation analysis» (besoins subjectifs et ressentis), et "present situation analysis» (l'acquis des apprenants). Ils y ajoutent les notions de considérations personnelles chez les apprenants (tradition culturelle, attitude, expériences en langues étrangères, etc.) et de facteurs environnementaux (contexte institutionnel ; voir également Holliday 1995 : 115-118). Enfin, J.C. Richards (2001: 53-4) conçoit également le terme "besoins» en tant que besoins actuels, potentiels et perçus, mais il insiste sur la notion d'une construction subjective - ou de représentation (cf. aussi Molinier 1996: chapitres 4-5, p. 147-148) -, fonction des intérêts et des valeurs personnels. Selon les acteurs, la perception des besoins peut évidemment varier considérablement.

5 Ensuite, comment sonder ces besoins? Plusieurs considérations méthodologiques influent sur la validité des résultats et donc sur l'impact de tout sondage en tant qu'outil d'aide à la décision: la définition précise de concepts et d'objectifs (Brown 2001: 1-29, Crosling \& Ward 2002: 42-45), les techniques de sondage et d'échantillonnage choisies (Brown 2001 : 30-70, voir également Dudley-Evans \& St. John 1998: 131-139, Richards 2001 : 59-63), le recueil et l'analyse des données (Brown 2001 : 71-252), et la manière dont les résultats sont présentés $(2001: 253-272)^{4}$. 


\subsection{Opérationnalisation}

6 Dans le but de définir la notion de maîtrise d'une langue étrangère pour la Faculté des Sciences économiques, pour mieux accorder l'offre d'enseignement à la demande, nous avons choisi de sonder quatre représentations, concernant les besoins cibles et les besoins d'apprentissage : le contexte d'utilisation de la (des) langue(s) étrangères(s) actuellement et surtout à l'avenir; les niveaux linguistiques estimés nécessaires pour la vie professionnelle et pour les études - tels qu'ils sont définis du débutant (A1) au très avancé (C2) par le Cadre européen commun de référence pour les langues (2001) - en matière de compréhension (lire, écouter) et de communication (écrire, parler); les attentes pédagogiques en matière d'enseignement et d'apprentissage linguistique à l'université ; l'importance des langues pour l'insertion professionnelle. Une dernière représentation sondée était celle de «l'image de soi » en tant qu'apprenant de langues, avec à la clé une auto-évaluation. Ce domaine personnel et affectif peut refléter en même temps une possible «programmation mentale » culturelle (Hofstede 1991:4) exerçant une influence sur les aspects cognitifs impliqués dans l'apprentissage des langues étrangères (EiseleHenderson $2001: 65$ ).

7 Ces différentes représentations des besoins ont été examinées dans quatre populations pour aborder la question de l'enseignement des langues du point de vue de toutes les catégories impliquées: étudiants, enseignants disciplinaires, enseignants de langues, diplômés. Pour atteindre un échantillon aussi large que possible, nous avons opté pour une enquête sous forme de questionnaire à questions fermées ${ }^{5}$, avec une invitation à ajouter tout commentaire voulu. Enfin, pour cerner le manque à gagner (compétences cibles moins compétences réelles), nous avons effectué un audit des compétences réceptives (lire, écouter, en anglais uniquement) ${ }^{6}$ basé sur les niveaux du Cadre européen commun de référence pour les langues (2001).

\subsection{Recueil des données}

Nos moyens humains et financiers nous ont permis d'administrer et de traiter, dans un premier temps (printemps 2003), le questionnaire pour les étudiants et celui destiné aux enseignants de Sciences économiques. L'enquête chez les enseignants de langues a été effectuée à l'automne 2003; celle chez les diplômés a été entreprise au printemps 2004 (son traitement est en cours au moment où nous écrivons). Les différentes versions du questionnaire ont été pilotées à l'automne 2003, et un texte d'accompagnement a été préparé avec le Doyen de la Faculté des Sciences économiques pour chaque public ciblé.

Nous avons choisi d'administrer le questionnaire aux étudiants du niveau Bac+2 (fin de l'enseignement obligatoire des langues) jusqu'au niveau Bac +5 inclus ${ }^{7}$. Atteindre près d'une vingtaine de groupes de TD de deuxième année en anglais, espagnol et allemand a exigé la participation de plusieurs collègues. Nous avons organisé une réunion pour leur présenter le protocole expérimental (questionnaire et, pour les groupes d'anglais, audit). L'administration chez les étudiants à partir de la troisième année a été faite par nousmême lors de cours disciplinaires en amphithéâtre (les enseignants nous ayant fait «don» du temps nécessaire, soit d'une heure et quart pour l'ensemble du protocole (questionnaire et audit), soit d'une vingtaine de minutes pour le questionnaire seul). 
10 Le questionnaire destiné aux enseignants titulaires d'économie et de langues a été diffusé par courrier interne à l'université, avec retour par la même voie. La version pour les diplômés a été adressée par courrier aux 795 diplômés de la maîtrise en Sciences économiques des promotions 1997-98, 1998-99 et 1999-2000.

\subsection{Analyse des données}

11 Cinquante pour cent de l'ensemble des étudiants de $\mathrm{Bac}+2$ à $\mathrm{Bac}+5$ ont répondu au questionnaire, soit 672 individus, répartis en Bac+2 ( $63 \%$ de la promotion), Bac+3 (51\%), $\mathrm{Bac}+4(26 \%)$ et Bac+5 (80\%). $41 \%$ de l'ensemble des étudiants ont participé à l'audit en anglais ( $72 \%$ des étudiants ayant choisi l'anglais comme langue obligatoire en Bac $+2,22 \%$ en Bac+3, $26 \%$ en Bac+4, $74 \%$ en Bac+5). Parmi les enseignants sondés, $38 \%$ ont rempli le questionnaire en Sciences économiques (30 individus) et $80 \%$ en langues (28 individus). À la date où nous écrivons, environ 230 réponses ont été recueillies parmi les diplômés, soit $29 \%$.

12 La saisie et le traitement des données pour les étudiants et les enseignants d'économie ont été assurés au printemps 2003 par deux étudiantes de l'IUP Ingénierie économique dans le cadre d'un stage professionnel de deux mois. Au moyen du logiciel de statistiques SAS, elles ont effectué le tri à plat (des pourcentages), des tris croisés (tableaux de contingence), ainsi que des analyses de la variance (ANOVA, pour savoir si tel facteur influe sur un autre, et si oui dans quelle mesure). Nous avons assuré nous-même le traitement des questionnaires des enseignants de langues (hiver 2004); celui des diplômés fait actuellement l'objet d'un nouveau stage professionnel pour trois étudiants de l'IUP.

\section{Résultats}

13 À partir de ces échantillons statistiquement représentatifs, des points clés marquants se dessinent pour chaque catégorie d'acteurs: étudiants, enseignants disciplinaires et enseignants de langues ${ }^{8}$. Nous traiterons, pour chaque groupe, les différentes représentations: contexte d'utilisation, niveaux estimés nécessaires, image de soi, attentes pédagogiques, ainsi que le manque à gagner (compétences cibles moins compétences réelles) chez les étudiants'. Là où c'est approprié, nous évoquerons certaines comparaisons entre les catégories d'acteurs.

\section{1. Étudiants}

14 Chez l'ensemble des étudiants, dont $29 \%$ sont étrangers, $67 \%$ disent utiliser une langue étrangère actuellement ( $90 \%$ l'anglais), et surtout pour leurs études (77\%). À l'avenir, $81 \%$ en prévoient une utilisation, surtout de l'anglais, pour le travail ; seulement $29 \%$ en prévoient dans le cadre des études, plutôt lire, écouter et parler qu'écrire. Les étudiants d'origine étrangère conçoivent une utilisation future plus importante que les Français (plus de $50 \%$ de plus pour les quatre compétences, et notamment pour la production écrite). Cette utilisation future est vue, pour $59 \%$ de tous les étudiants, ailleurs qu'en France avec des locuteurs natifs ; seulement 39 \% imaginent utiliser une langue étrangère en France avec des locuteurs non natifs (y compris des Français). De manière significative dans tous les cas, les étudiants expriment davantage de difficultés par rapport aux 
compétences qu'ils estiment les plus importantes professionnellement, respectivement communiquer (écouter $34 \%$, parler $31 \%$ ) et comprendre (écrire $23 \%$, lire $18 \%$ ).

Quant aux niveaux linguistiques estimés nécessaires pour la vie professionnelle et pour les études, la représentation pour le travail - maximale partout - est supérieure à celle pour les études (Tableaux 1.a-1.d). Écouter (surtout C2) devance les trois autres compétences, qui sont à égalité pour la vie professionnelle, alors que lire-écrire (surtout C1-B2) sont jugés plus importants qu'écouter-parler pour les études (B2-B1). Les étrangers accordent toujours une plus grande importance que les Français aux quatre compétences, à la fois pour le travail et pour les études. Enfin, si $41 \%$ de l'échantillon expriment une demande de reconnaissance externe (certification) de leur niveau en langue étrangère du type examens de Cambridge ou TOEFL, la distinction entre Français et étrangers reste significative, respectivement $35 \%$ et $66 \%$.

Tableau 1 - Représentations des besoins cibles par compétence linguistique et par catégorie d'acteurs (d'après le Conseil de l'Europe)

\section{A1 Débutant (utilisateur de base) \\ A2 Élémentaire (utilisateur de base) \\ B1 Intermédiaire faible (utilisateur indépendant) \\ B2 Intermédiaire fort (utilisateur indépendant) \\ C1 Avancé (utilisateur expérimenté) \\ C2 Très avancé (utilisateur expérimenté)}

a. Lire

\begin{tabular}{|l|l|l|l|l|}
\hline & $\begin{array}{l}\text { Ets. } \\
\text { études } \\
(\mathrm{n}=672)\end{array}$ & $\begin{array}{l}\text { Ets. } \\
\text { travail } \\
(\mathrm{n}=672)\end{array}$ & $\begin{array}{l}\text { Ens. } \\
\text { Éco. } \\
(\mathrm{n}=30)\end{array}$ & $\begin{array}{l}\text { Ens. } \\
\text { Langues } \\
(\mathrm{n}=28)\end{array}$ \\
\hline C2 & & $34 \%$ & & \\
\hline C1 & $(21 \%)$ & $31,4 \%$ & $36,7 \%$ & $51,6 \%$ \\
\hline B2 & $31,7 \%$ & & $40 \%$ & $45,2 \%$ \\
\hline B1 & $(19 \%)$ & & & \\
\hline A2 & & & & \\
\hline A1 & & & & \\
\hline
\end{tabular}

\section{b. Écrire}

\begin{tabular}{|l|l|l|l|l|}
\hline & $\begin{array}{l}\text { Ets. } \\
\text { études } \\
(\mathrm{n}=672)\end{array}$ & $\begin{array}{l}\text { Ets. } \\
\text { travail } \\
(\mathrm{n}=672)\end{array}$ & $\begin{array}{l}\text { Ens. } \\
(\mathrm{n}=30)\end{array}$ & $\begin{array}{l}\text { Ens. } \\
\text { Langues } \\
(\mathrm{n}=28)\end{array}$ \\
\hline $\mathrm{C} 2$ & & $33,9 \%$ & & \\
\hline
\end{tabular}




\begin{tabular}{|l|l|l|l|l|}
\hline C1 & $(18,6 \%)$ & $29,5 \%$ & & $(16,1 \%)$ \\
\hline \hline B2 & $32,9 \%$ & & $46,7 \%$ & $58,1 \%$ \\
\hline B1 & $(18,1 \%)$ & & $(20 \%)$ & \\
\hline \hline A2 & & & & \\
\hline \hline A1 & & & & \\
\hline
\end{tabular}

\section{c. Écouter}

\begin{tabular}{|l|l|l|l|l|}
\hline & $\begin{array}{l}\text { Ets. } \\
\text { études } \\
(\mathrm{n}=672)\end{array}$ & $\begin{array}{l}\text { Ets. } \\
\text { travail } \\
(\mathrm{n}=672)\end{array}$ & $\begin{array}{l}\text { Ens. } \\
\text { Éco. } \\
(\mathrm{n}=30)\end{array}$ & $\begin{array}{l}\text { Lans. } \\
(\mathrm{n}=28)\end{array}$ \\
\hline C2 & & $43 \%$ & & \\
\hline C1 & $(14,1 \%)$ & $(19 \%)$ & & $(22,6 \%)$ \\
\hline B2 & $27,7 \%$ & & $26,7 \%$ & $67,7 \%$ \\
\hline B1 & $27,2 \%$ & & $33,3 \%$ & \\
\hline A2 & & & & \\
\hline A1 & & & & \\
\hline
\end{tabular}

\section{d. Parler}

\begin{tabular}{|l|l|l|l|l|}
\hline & $\begin{array}{l}\text { Ets. } \\
\text { études } \\
(\mathrm{n}=672)\end{array}$ & $\begin{array}{l}\text { Ets. } \\
\text { travail } \\
(\mathrm{n}=672)\end{array}$ & $\begin{array}{l}\text { Ens. } \\
\text { Éco. } \\
(\mathrm{n}=30)\end{array}$ & $\begin{array}{l}\text { Ens. } \\
\text { Langues } \\
(\mathrm{n}=28)\end{array}$ \\
\hline C2 & & $32,3 \%$ & & \\
\hline C1 & & $29,3 \%$ & & $(32,3 \%)$ \\
\hline B2 & $26 \%$ & & $30 \%$ & $54,8 \%$ \\
\hline B1 & $28,7 \%$ & & $40 \%$ & \\
\hline \hline A2 & & & & \\
\hline A1 & & & & \\
\hline
\end{tabular}


Quand on compare la représentation des niveaux cibles nécessaires aux compétences réelles telles qu'elles ont été mesurées (Tableaux 2.a-2.d), le manque à gagner se révèle important, surtout au niveau de la compréhension de texte. L'image qu'ont les étudiants d'eux-mêmes en tant qu'apprenants de langues s'en ressent : l'auto-évaluation du niveau actuel des quatre compétences est en dessous des niveaux cibles. Ils s'estiment meilleurs en lecture que, respectivement, en écriture, en écoute et en expression orale. Les étrangers, encore une fois, accordent une plus grande importance que les Français aux quatre compétences pour les études et pour le travail, et s'évaluent à des niveaux plus élevés. À un niveau légèrement moindre que les Français (79 \% contre $89 \%$ ), ils partagent néanmoins avec leurs hôtes la perception que « les Français sont mauvais en langues ».

Tableau 2 - Étudiants : représentation des niveaux cibles par compétence linguistique, autoévaluation, audit (lire, écouter)

a. Lire

\begin{tabular}{|l|l|l|l|l|}
\hline & $\begin{array}{l}\text { Cibles } \\
\text { études } \\
(\mathrm{n}=672)\end{array}$ & $\begin{array}{l}\text { Cibles } \\
\text { travail } \\
(\mathrm{n}=672)\end{array}$ & $\begin{array}{l}\text { Auto-évaluation } \\
(\mathrm{n}=672)\end{array}$ & $\begin{array}{l}\text { Audit } \\
(\mathrm{n}=460)\end{array}$ \\
\hline C2 & & $34 \%$ & & \\
\hline C1 & $(21 \%)$ & $31,4 \%$ & & \\
\hline B2 & $31,7 \%$ & & $34,1 \%$ & $36,8 \%$ \\
\hline B1 & $(19 \%)$ & & $25,5 \%$ & $47,7 \%$ \\
\hline \hline A2 & & & $(19,5 \%)$ & \\
\hline A1 & & & & \\
\hline
\end{tabular}

b. Écrire

\begin{tabular}{|l|l|l|l|}
\hline & $\begin{array}{l}\text { Cibles } \\
\text { études } \\
(\mathrm{n}=672)\end{array}$ & $\begin{array}{l}\text { Cibles } \\
\text { travail } \\
(\mathrm{n}=672)\end{array}$ & $\begin{array}{l}\text { Auto-évaluation } \\
(\mathrm{n}=672)\end{array}$ \\
\hline C2 & & $33,9 \%$ & \\
\hline C1 & $(18,6 \%)$ & $29,5 \%$ & \\
\hline B2 & $32,9 \%$ & & $(16,5 \%)$ \\
\hline B1 & $(18,1 \%)$ & & $44,3 \%$ \\
\hline A2 & & & $(15,8 \%)$ \\
\hline
\end{tabular}


A1

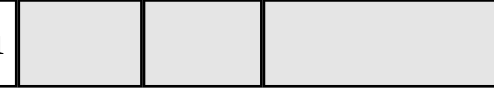

c. Écouter

\begin{tabular}{|l|l|l|l|l|}
\hline & $\begin{array}{l}\text { Cibles } \\
\text { études } \\
(\mathrm{n}=672)\end{array}$ & $\begin{array}{l}\text { Cibles } \\
\text { travail } \\
(\mathrm{n}=672)\end{array}$ & $\begin{array}{l}\text { Auto-évaluation } \\
(\mathrm{n}=672)\end{array}$ & $\begin{array}{l}\text { Audit } \\
(\mathrm{n}=460)\end{array}$ \\
\hline C2 & & $43 \%$ & & \\
\hline C1 & $(14,1 \%)$ & $(19 \%)$ & & \\
\hline B2 & $27,7 \%$ & & & $36,8 \%$ \\
\hline B1 & $27,2 \%$ & & $44,7 \%$ & $47,7 \%$ \\
\hline A2 & & & $(20,7 \%)$ & \\
\hline A1 & & & $(15,7 \%)$ & \\
\hline
\end{tabular}

d. Parler

\begin{tabular}{|l|l|l|l|}
\hline & $\begin{array}{l}\text { Cibles } \\
\text { études } \\
(\mathrm{n}=672)\end{array}$ & $\begin{array}{l}\text { Cibles } \\
\text { travail } \\
(\mathrm{n}=672)\end{array}$ & $\begin{array}{l}\text { Auto-évaluation } \\
(\mathrm{n}=672)\end{array}$ \\
\hline C2 & & $32,3 \%$ & \\
\hline C1 & & $29,3 \%$ & \\
\hline B2 & $26 \%$ & & \\
\hline \hline B1 & $28,7 \%$ & & $22,5 \%$ \\
\hline A2 & & & $29 \%$ \\
\hline A1 & & & $27,8 \%$ \\
\hline
\end{tabular}

17 Pour remédier à cette situation, au niveau des attentes pédagogiques, on observe une forte demande d'un enseignement linguistique obligatoire (93\%), pour une seule langue et sans interruption jusqu'au Bac +5 (66 \% pour l'ensemble, $82 \%$ en DESS). $34 \%$ souhaitent l'étude obligatoire (par rapport à une offre facultative) d'une deuxième langue étrangère. Pour $55 \%$ des étudiants, trois à quatre heures par semaine représentent un investissement raisonnable dans l'étude de la langue principale. Toutefois, une certaine résistance au changement se manifeste: $34 \%$ sont favorables à un enseignement présentiel, mais seulement $7,5 \%$ au travail en autonomie guidée (éventuellement en 
ligne). $55 \%$, néanmoins, envisageraient un mélange de ces deux modes de travail. Enfin, l'attente est forte d'une intégration de langue(s) étrangère(s) dans des matières non linguistiques : $60 \%$ à Bac+2 et 3, mais $81 \%$ à Bac +4 et $90 \%$ à Bac+5.

Invités à formuler des commentaires libres, de nombreux étudiants en Sciences économiques se sont plaints de leur faible niveau en langues après plusieurs années d'études, et ont dit estimer que leur niveau à l'université a baissé depuis le lycée. Ils se sentent frustrés de ne pas pouvoir améliorer la situation (hors mobilité internationale), et disent se sentir défavorisés par rapport à leurs camarades étrangers qui élèvent le niveau et font baisser les moyennes générales.

Ces perceptions ne sont, d'ailleurs pas infondées : les résultats de l'audit s'expliquent de manière statistiquement significative par un certain nombre de variables. Le score en lecture diffère, par exemple, en fonction de la nationalité (les étrangers étant meilleurs que les Français), d'une pratique plus fréquente de la lecture et de l'écriture, et d'une auto-évaluation (et donc de l'image de soi en tant qu'apprenant des langues) plus élevée en lecture comme en écriture. De la même manière, le score en compréhension auditive diffère significativement en fonction de l'année d'études (après la dernière année d'étude obligatoire de langue en $\mathrm{Bac}+2$, baisse progressive en $\mathrm{Bac}+3$ et $\mathrm{Bac}+4$, avec une remontée en $\mathrm{Bac}+5$, mais dans la population extrêmement sélective d'étudiants en DESS, Tableau $3 b$ ), d'une pratique plus fréquente de l'interaction orale (écouter, parler), et d'une autoévaluation plus élevée pour les deux compétences de l'interaction orale.

Tableau 3 - Audit ( $n=460)$ : pourcentages par niveaux de compétence (A2-B2) par année

a. Lire

\begin{tabular}{|l|l|l|l|l|}
\hline & $\begin{array}{l}\mathrm{Bac}+2 \\
(\mathrm{n}=215)\end{array}$ & $\begin{array}{l}\mathrm{Bac}+3 \\
(\mathrm{n}=76)\end{array}$ & $\begin{array}{l}\mathrm{Bac}+4 \\
(\mathrm{n}=101)\end{array}$ & $\begin{array}{l}\mathrm{Bac}+5 \\
(\mathrm{n}=67)\end{array}$ \\
\hline \hline B2 & $6,5 \%$ & $3,9 \%$ & $9,9 \%$ & $13,4 \%$ \\
\hline B1 & $34,3 \%$ & $44,7 \%$ & $30,7 \%$ & $46,3 \%$ \\
\hline A2 & $49,8 \%$ & $44,7 \%$ & $54,5 \%$ & $34,3 \%$ \\
\hline
\end{tabular}

b. Écouter

\begin{tabular}{|l|l|l|l|l|}
\hline & $\begin{array}{l}\mathrm{Bac}+2 \\
(\mathrm{n}=215)\end{array}$ & $\begin{array}{l}\mathrm{Bac}+3 \\
(\mathrm{n}=76)\end{array}$ & $\begin{array}{l}\mathrm{Bac}+4 \\
(\mathrm{n}=101)\end{array}$ & $\begin{array}{l}\mathrm{Bac}+5 \\
(\mathrm{n}=67)\end{array}$ \\
\hline B2 & $6,6 \%$ & $11,8 \%$ & $5,9 \%$ & $20,9 \%$ \\
\hline B1 & $41,8 \%$ & $34,2 \%$ & $29,7 \%$ & $41,8 \%$ \\
\hline A2 & $43,7 \%$ & $48,7 \%$ & $57,4 \%$ & $28,4 \%$ \\
\hline
\end{tabular}




\subsection{Enseignants en Sciences économiques}

20 Chez les enseignants disciplinaires, le sondage avait un double objectif : d'abord cerner la notion de maîtrise (ou du moins le niveau nécessaire) des compétences en langues chez des étudiants à $\mathrm{Bac}+5$, et deuxièmement, refléter, en tant que catégorie professionnelle ${ }^{10}$ et membres de la communauté universitaire, leur utilisation des langues étrangères et leurs propres difficultés. Dans le cas présent, c'est le premier de ces objectifs qui nous concerne; nous évoquerons uniquement les représentations des niveaux estimés nécessaires chez les étudiants et les attentes pédagogiques à l'égard de ces derniers.

Ainsi, les enseignants d'économie sont moins exigeants que les étudiants par rapport aux niveaux de compétence à atteindre au stade de $\mathrm{Bac}+5$, celui de la fin des études et de l'entrée dans la vie professionnelle. Si un niveau B2-C1 suffit en lecture (pour l'anglais à $97 \%$ ), B1-B2 convient pour les autres compétences (Tableaux 1.a-1.d). Comme les étudiants dans le cadre universitaire (mais non pour la vie professionnelle), les enseignants estiment que lire en langue étrangère est la compétence la plus importante, suivie d'écrire (Tableaux 1.a-1.d). Ils sont plus nombreux que leurs étudiants à sentir qu'une certification en compétence linguistique est importante ( $78 \%$ contre $41 \%$ ).

Globalement, les étudiants sélectionnés en DESS se rapprochent le plus des niveaux souhaités par leurs enseignants en écoute et en lecture (Tableaux 1.a-1.d, 3a-3.b), mais la performance des étudiants telle que mesurée n'atteint pas le but visé. Comme les étudiants d'ailleurs, les enseignants répondent massivement (92\%) que «les Français (parmi lesquels ils se comptent) sont mauvais en langues ", bien qu'ils s'estiment plutôt compétents (surtout pour lire, $60 \% \mathrm{C} 2-\mathrm{C} 1,23 \%$ B2) dans leur vie professionnelle (écrire $73 \%$ B2-C1, parler $63 \%$ B2-C2, écouter $60 \%$ B2-C2).

En ce qui concerne les attentes pédagogiques à l'égard des étudiants, les enseignants d'économie prônent très fortement $(90 \%)$ l'étude obligatoire d'une (seule) langue étrangère dans le cursus universitaire ; seulement 17 \% envisagent favorablement l'étude obligatoire (et non facultative) d'une deuxième langue. L'attente d'une intégration de langue(s) dans des matières disciplinaires est également marquée ( $80 \%$, mais selon la situation d'apprentissage: lectures en langue étrangère (79\%), cours magistraux/TD (58\%), présentations orales ou exposés de la part des étudiants (50\%), mais seulement $38 \%$ sont favorables aux productions écrites (mémoires, rapports de stage, examens).

\subsection{Enseignants de langues}

Au niveau des compétences estimées nécessaires à Bac +5 , les enseignants de langues sont plus exigeants que les enseignants disciplinaires, mais toujours moins que les étudiants vis-à-vis d'eux-mêmes. Ils pensent que lire est plus important (C1-B2) que parler et écouter (B2-C1) et écrire (B2, Tableaux 1.a-1.d). Plus près des enseignants disciplinaires que les étudiants, les enseignants de langues sont $71 \%$ (contre respectivement $78 \%$ et $41 \%$ ) à penser que les étudiants devraient obtenir une certification en compétence linguistique.

Leur degré de satisfaction par rapport au niveau linguistique des étudiants en Sciences économiques est, sans surprise, plus important au fur et à mesure que le niveau d'études - et de langue de spécialité - monte ${ }^{11}$. Par conséquent, les attentes pédagogiques sont clairement exprimées, de manière à mieux préparer les étudiants à progresser. Tout 
d'abord, $79 \%$ des enseignants de langues souhaiteraient voir définir une progression d'objectifs à partir de Bac+1, basée sur le Cadre européen commun de référence pour les langues. Presque tous insistent sur l'étude obligatoire d'au moins une langue, avec entre trois et quatre heures d'investissement présentiel et personnel par semaine (83\%). Avec 27 \% favorables à l'étude obligatoire d'une deuxième langue, les enseignants de langues sont davantage demandeurs que les enseignants disciplinaires (17\%) mais moins que les étudiants (33\%). 90 \% des répondants prônent l'enseignement de la langue de spécialité, majoritairement à partir de $\mathrm{Bac}+3$.

Chez ces enseignants, comme chez leurs collègues en Sciences économiques et chez les étudiants, l'attente est forte d'une intégration de langue(s) étrangère(s) dans des matières disciplinaires ( $86 \%$ ): $77 \%$ pour les présentations orales ou exposés de la part des étudiants, $60 \%$ pour la lecture, $60 \%$ pour les $\mathrm{CM} / \mathrm{TD}$, et, exactement comme leurs collègues en économie, $38 \%$ pour les productions écrites. Pour mener à bien cette intégration, $90 \%$ conçoivent travailler en équipe avec leurs collègues en sciences économiques ${ }^{12}$. Enfin, une demande forte est exprimée de pouvoir travailler autrement: tout en maintenant un enseignement présentiel (77\%), celui-ci doit se faire par groupes de niveau $(77 \%)$. La possibilité de travailler en autonomie guidée est également souhaitée par $71 \%$, mais seulement $48 \%$ sont favorables au travail par projets avec équipes d'étudiants.

\section{Discussion}

En attendant les résultats du sondage chez les diplômés, on voit déjà que, chez les différents acteurs, les diverses compétences linguistiques jouissent d'une importance variable. Les étudiants, pour leurs études, attachent davantage de poids à l'écrit (lire, écrire, C1-B2) qu'à l'oral (écouter-parler, B2-B1). Mais pour leur futur travail, le niveau maximal est majoritairement estimé nécessaire, surtout pour écouter (C2). Les deux catégories d'enseignants ciblent des niveaux probablement plus réalistes, plus proches de ceux que ciblent les étudiants pour le cadre universitaire. La lecture est classée comme la plus importante (C1-B2), suivie, pour les économistes, par écrire et écouter-parler (B2), et, pour les linguistes, pour l'oral (B2-C1) et la production écrite (B2).

Qu'est-ce donc que la notion de «maitrise » d'une langue étrangère dans l'enseignement universitaire des Sciences économiques? Quels sont les besoins cibles? Mise à part la représentation professionnelle chez les étudiants (que nous qualifierons de trop perfectionniste), la lecture est donc majoritairement ressentie par les différents acteurs comme étant la compétence la plus importante, et ceci au niveau défini par le Conseil de l'Europe C1-B2, avancé-intermédiaire fort. Pour les autres compétences, le classement des représentations diverge, mais se situe aux niveaux B2-B1, avec les deux compétences orales à des taux plus forts.

La réponse à notre question n'est donc pas absolue, mais dépend de la catégorie de répondants. Le témoignage des diplômés complétera de manière indispensable le tableau. Mais à court terme, pour que l'offre réponde mieux à la demande nouvelle qu'implique le master, on doit prendre en compte également la différence entre besoins cibles et compétences réelles (le manque à gagner), l'image de soi des apprenants, et les besoins d'apprentissage tels qu'ils sont exprimés. 
30 L'audit pratiqué en lecture et en compréhension auditive a révélé que les étudiants sont nettement en dessous des niveaux cibles, et leur auto-évaluation montre qu'ils en sont conscients, surtout au niveau professionnel. Le manque à gagner est donc réel et est perçu comme important.

31 Il est critique également sous un autre angle: les étudiants font état de plus grandes difficultés par rapport aux compétences orales, qu'ils estiment les plus importantes pour la vie professionnelle. En même temps les enseignants privilégient la lecture au niveau Bac+5. Nous avons vu que les deux groupes sont favorables à l'intégration d'une langue étrangère dans des matières non linguistiques. Mais cette intégration est conçue sous forme de lectures, de cours/TD et de présentations orales faites par les étudiants, alors que les résultats de l'audit en lecture et en compréhension auditive situent près de la moitié de l'ensemble des étudiants au niveau élémentaire et seulement un tiers au niveau intermédiaire faible (Tableau 2). Et par année d'études, si les Tableaux $3(a-b)$ et 1 (a-d) montrent que les étudiants en $\mathrm{Bac}+5$ se rapprochent le plus des niveaux cibles souhaités par leurs enseignants, les $\mathrm{Bac}+4$ (les futurs master première année) sont les plus faibles à tous les niveaux.

Nous avons aussi vu que les étudiants sont trèsdemandeurs et motivés pour améliorer leur niveau en langue(s) étrangère(s), disant vouloir y consacrer plusieurs heures par semaine, de manière régulière, voire de manière intégrée à leurs matières disciplinaires. Dans leurs commentaires, ils insistent particulièrement sur l'égalité des chances, sur un coefficient plus valorisant dans leur diplôme (voire l'obligation d'un niveau seuil de compétence obligatoire à atteindre), sur une plus grande possibilité de mobilité, sur des préparations ciblées pour des concours, de la recherche, etc. Certains étudiants plaident pour le plurilinguisme, comme cette étudiante de maîtrise : «On construit l'Europe économique, mais quel retard dans la construction d'une Europe des peuples! Ce n'est pas seulement l'anglais qu'il faut apprendre, mais les autres grandes langues. Pour y arriver, il faut s'en donner les moyens et renforcer l'apprentissage ».

\section{Conclusions : recommandations, vers une réelle politique linguistique?}

Ces résultats nous ont permis de proposer plusieurs pistes à la Faculté des Sciences économiques pour que l'offre réponde à la demande, même si l'on sait qu'il y aura toujours un fossé entre le devoir faire et le pouvoir faire (notamment en ce qui concerne l'augmentation du volume horaire des cours de langues). Il est d'abord fondamental, à partir de l'analyse des questionnaires, que les enseignants disciplinaires comprennent l'importance de définir des objectifs linguistiques critériés : que tel niveau de compétence en lecture, en expression orale, etc., est nécessaire (ou fortement conseillé) pour tel diplôme selon son niveau (licence, master) et son orientation scientifique et professionnelle; que ces objectifs soient clairement explicités aux étudiants; qu'ils correspondent de manière transparente à un nombre de « crédits » appropriés. En même temps, à partir d'exemples de bonnes pratiques, nous encourageons la réflexion sur la spécificité de l'apprentissage des langues par les spécialistes d'autres disciplines.

Afin de bâtir une offre renouvelée d'enseignement des langues, un redéploiement institutionnel des moyens financiers, matériels et humains est également proposé. En accord avec l'arrêté de la licence qui prévoit une évaluation des étudiants ${ }^{13}$ et la mise en 
place d'un enseignement approprié, nous prévoyons, par exemple, des groupes de niveau pour les cours présentiels pour la rentrée 2004. En parallèle, la création d'un centre de langues digne de ce nom fait partie du projet à plus long terme; en regroupant l'ensemble des moyens consacrés aux langues (actuellement dispersés par composantes disciplinaires) et en mutualisant les ressources en nouvelles technologies, elle permettra de favoriser l'apprentissage en autonomie guidée. À plus court terme, nous jouons également un rôle de médiatrice entre les enseignants disciplinaires et les enseignants de langues pour encourager la collaboration nécessaire à une intégration réussie (cours enseignés en langue étrangère, cf. Marsh, Marsland \& Stenberg 2001 ; Snow \& Brinton 1997 ; Wolff, à paraître).

Une dernière innovation pédagogique proposée est l'utilisation à grande échelle du Portfolio européen des langues. Cet outil d'auto-évaluation basé sur une approche métacognitive - apprendre à apprendre - est, de notre point de vue, la meilleure manière d'encourager les étudiants à prendre en charge leur propre apprentissage dans le contexte universitaire (Little \& Perclová 2001). Aider l'apprenant à établir des objectifs réalistes et à expliciter les moyens nécessaires pour y arriver, ainsi que l'échéance appropriée, nous paraît la manière la plus efficace de combattre les représentations exagérément ambitieuses, et donc irréalisables et frustrantes, telles que celles définies comme besoins cibles pour le travail par les étudiants de notre échantillon.

Cette analyse des besoins en langues étrangères pour la Faculté des Sciences économiques a donc eu un impact immédiat et concret en tant qu'outil d'aide à la décision : la demande des acteurs, une fois connue, a été entendue et des réponses sont à l'étude. Certaines innovations sont déjà programmées, et nous attendons davantage de précisions des résultats du sondage chez les diplômés pour guider la réflexion pour l'avenir. Mais, dès à présent, l'évaluation de toute innovation mise en œuvre - que cela soit au niveau des objectifs linguistiques définis, au niveau des modes de fonctionnement, etc. - est impérative, dans un souci de transparence et de confiance mutuelle entre enseignants de langues et responsables disciplinaires et universitaires.

Cette enquête a eu une autre retombée inattendue et positive. Grâce à la réflexion ouverte dans une Faculté sur la place des langues dans le LMD, nous avons été sollicitée pour coordonner la même réflexion pour l'ensemble de la communauté universitaire. Cette tâche est par définition transversale, amenant obligatoirement à collaborer avec le Département des langues, toutes les filières disciplinaires, la scolarité, le service informatique (pédagogique et réseau), le bureau de la mobilité étudiante et les bibliothèques universitaires. La démarche, déjà entamée, est l'extension de celle déjà amorcée en Sciences économiques: définition des objectifs par sondage auprès des responsables et des enseignants de chaque filière (et par la même occasion, sensibilisation à la spécificité de l'apprentissage des langues), illustration d'exemples de bonnes pratiques, propositions pédagogiques à court et à plus long terme, évaluation de toute approche mise en œuvre.

38 Cette sollicitation, par la reconnaissance qu'elle donne aux langues étrangères dans une université spécialisée dans d'autres disciplines, constitue un premier pas vers une réelle politique linguistique locale. C'est un défi qui implique un changement de mentalités et de culture universitaire; nous ne pouvons qu'espérer que «langue étrangère » ne soit plus synonyme de « langue de bois ». 
Nous tenons à remercier Jacques LePottier, Doyen de la Faculté des Sciences économiques de l'Université Toulouse 1 Sciences Sociales, pour sa collaboration et son soutien; Michel Bonneu, pour son co-encadrement des étudiants stagiaires ; les stagiaires elles-mêmes (Sandra Dieudé et Alix Delvillar), pour leur sérieux; Marc Boudier, de l'Observatoire de la vie étudiante, pour ses conseils en matière de sondage; ainsi que les lecteurs du comité de lecture pour leurs suggestions.

\section{BIBLIOGRAPHIE}

Brown, J. D. 2001. Using Surveys in Language Programs. Cambridge : Cambridge University Press.

Conseil de l'Europe.2001. Cadre européen commun de référence pour les langues : apprendre, enseigner, évaluer. Paris : Didier.

Crosling, G. \& I. Ward. 2002. « Oral communication : The workplace needs and uses of business graduate employees ». English for Specific Purposes 21, 41-57.

Dudley-Evans, T. \& M. J. St. John. 1998. Developments in English for Specific Purposes: A Multidisciplinary Approach. Cambridge : Cambridge University Press.

Eisele-Henderson, A. 2001. La Lecture de textes en anglais langue étrangère chez des étudiants en sociologie : implications didactiques d'une perspective socio-cognitive. Thèse de doctorat, Université de Savoie.

Hofstede, G. 1991. Cultures and Organizations: Software of the Mind. Cambridge : McGraw-Hill International.

Holliday, A. 1995. « Assessing language needs within an institutional context : An ethnographic approach ». English for Specific Purposes 14, 115-126.

Hutchinson, T. \& A. Waters. 1987. English for Specific Purposes: A Learning-centred Approach. Cambridge : Cambridge University Press.

Little, D. \& R. Perclova. 2001. Portfolio européen des langues. Guide à l'usage des enseignants et formateurs d'enseignants. Strasbourg : Conseil de l'Europe <http://culture2.coe.int/portfolio>.

Marsh, D., B. Marsland \& K. Stenberg. 2001. Integrating Competencies for Working Life. Jyväskylä, Finlande : Unicom, University of Jyväskylä.

Molinier, P. 1996. Images et représentations sociales: de la théorie des représentations à l'étude des images sociales. Grenoble : Presses universitaires de Grenoble.

Richards, J. C. 2001. Curriculum Development in Language Teaching. Cambridge : Cambridge University Press.

Snow, M. A. \& D. M. Brinton (dir). 1997. The Content-based Classroom: Perspectives on Integrating Language and Content. White Plains, NY : Longman.

Taillefer, G. 1992. Les Difficultés de lecture de l'anglais, langue étrangère, chez des étudiants en sciences sociales. Thèse de doctorat, Université de Toulouse-Le Mirail.

Wolff, D. à paraître. « Integrating language and content in the language classroom: Are transfer of knowledge and of language ensured ? ». 24 e colloque du GERAS, 20-22 mars, 2003, Le Havre. 


\section{NOTES}

1. Arrêté du 25 avril 2002, J.O. n 99 du 27 avril 2002, p. 7631.

2. UT1 Magazine, $n^{\circ} 81$ bis, septembre 2003.

3. La recherche en Sciences économiques effectuée à l'Université des Sciences sociales Toulouse 1 est classée au premier rang en Europe. Voir «Universités : le palmarès 2003 ». Le Nouvel Observateur 13-19 mars 2003, 14-54.

4. J. C. Richards (2001: 63-64, 72-73) propose une liste succincte récapitulative des considérations principales.

5. Le questionnaire destiné aux étudiants est fourni en Annexe ; les versions respectives pour les deux groupes d'enseignants et pour les diplômés sont disponibles auprès de l'auteure.

6. Oxford Quick Placement Test pour la compréhension de textes, la grammaire et le vocabulaire ; Oxford Placement Test, Listening pour la compréhension auditive.

7. DESS uniquement, les DEA étant enseignés complètement en anglais.

8. Des résultats détaillés sont disponibles auprès de l'auteure.

9. La représentation de l'importance des langues pour l'insertion professionnelle est traitée de manière spécifique dans le sondage destiné aux diplômés. Au moment où nous écrivons, ces données ne sont pas encore disponibles.

10. Certains étudiants, bien que le pourcentage soit faible d'après l'enquête OVE citée ci-dessus, poursuivent cette voie professionnelle. Les résultats seront à comparer avec ceux des diplômés.

11. Le degré de satisfaction à l'égard des étudiants est similaire pour les étudiants en Sciences économiques et en Droit, mais il est plus important que pour la filière AES, de série de Baccalauréat et d'origine sociale généralement différents des deux premières filières (Taillefer 1992).

12. Malheureusement, nous n'avons pas posé cette question dans l'autre sens aux enseignants d'économie : concevez-vous de travailler en équipe avec des enseignants de langues?

13. Arrêté du 23 avril 2002, J.0. nº101 du 30 avril 2002, p. 7821. Nous prévoyons d'utiliser le test DIALANG <http://www.dialang.org>.

\section{RÉSUMÉS}

Comment l'enseignement des langues dans le secteur LANSAD peut-il répondre aux niveaux de compétences linguistiques exigés par des milieux professionnels et au nouveau diplôme de master exigeant du candidat la maitrise d'au moins une langue étrangère ? Pour répondre à ces questions, une analyse des besoins a été effectuée, basée sur la grille des compétences linguistiques du Conseil de l'Europe. Un questionnaire a été administré à des étudiants, des enseignants et des diplômés en Sciences économiques, ainsi qu'à des enseignants de langues. Les représentations sondées étaient le contexte d'utilisation de la langue, les niveaux cibles des différentes compétences, l'auto-évaluation et les attentes pédagogiques, et l'importance des langues pour l'insertion professionnelle. Un audit a également été pratiqué (lire, écouter) chez les étudiants. L'image diffère selon les échantillons : si les enseignants perçoivent des besoins cibles « raisonnables", les étudiants visent une compétence professionnelle maximale. Mais ils s'estiment faibles (et le sont, d'après l'audit). D'où beaucoup d'attentes exprimées, mais aussi 
l'ouverture d'une réflexion et d'un engagement politique et pédagogique, faisant de l'outil de recherche une aide à la décision.

How can LSP teaching meet the target language competency needs of professionals as well as those of the new French master degree requiring mastery of at least one foreign language? To answer these questions, a needs analysis was carried out, based on the Council of Europe's language competency grid. A questionnaire was given to students, teachers and graduates in Economics, as well as to university language teachers. Perceptions explored were the context of language use, the target levels of the different competencies, self-assessment and learner needs, and the importance of languages for the job market. A language audit was also carried out (reading, listening comprehension) among students. Perceptions differ among groups of stakeholders: if teachers perceive a "reasonable" level of target needs, students feel that they will need maximum professional language competency. But their self-assessment is weak - a perception borne out by the audit. Consequently, many learner needs are expressed, at the same time as institutional discussion of language policy and pedagogy have opened up. The research questionnaire has thus become a decision-making tool.

\section{INDEX}

Mots-clés : analyse des besoins, attente pédagogique, compétence cible, outil d'aide à la décision, représentation

Keywords : decision-making tool, learner need, needs analysis, perception, target competency

\section{AUTEUR}

\section{GAIL TAILLEFER}

